\title{
IMPLANTAÇÃO DO SISTEMA INTEGRADO DE GESTÃO DE LABORATÓRIOS DE PESQUISA: UM RELATO DA EXPERIÊNCIA NA UFABC
}

\section{IMPLEMENTING THE INTEGRATED MANAGEMENT SYSTEM FOR THE RESEARCH LABS: UFABC' EXPERIENCE REPORT}

\section{IMPLEMENTACIÓN DEL SISTEMA INTEGRADO DE GESTIÓN DE LABORATORIOS DE INVESTIGACIÓN: UN RELATO DE LA EXPERIENCIA EN UFABC}

Artur Leonardo Imamura Ferreira da Silva.

Mestre em Gestão de Políticas e Organizações Públicas pela Universidade Federal de São Paulo. Doutorando em Administração pela Universidade Municipal de São Caetano do Sul turimamura@yahoo.com.br https://orcid.org/0000-0002-0451-3132

Keilla Dayane da Silva Oliveira

Mestrado em Ciência, Tecnologia e Ambiente pela UFVJM. Doutoranda do Programa de Pós Graduação em Administração - USCS

keilladsoliveira@gmail.com

https://orcid.org/0000-0003-0018-2484

Alyne Mantoan, Isabel Cristina dos Santos

Técnico-Administrativo na Universidade Municipal de São Caetano do Sul. Mestranda do Programa de Pós Graduação em Administração - USCS

alynemantoan@gmail.com

https://orcid.org/0000-0002-5611-6438

Isabel Cristina dos Santos

Docente do Programa de Pós-graduação em Administração da Universidade Municipal De São Caetano do Sul - USCS isabel.santos@prof.uscs.edu.br http://orcid.org/0000-0001-5505-5234

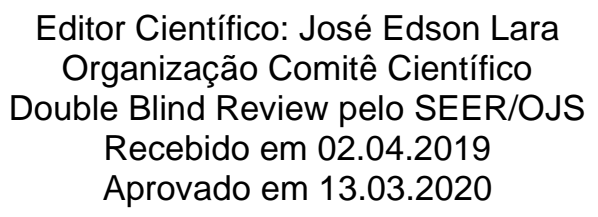




\title{
Resumo
}

Objetivo: descrever como o processo de implantação do sistema integrado de gestão de laboratórios de pesquisa, pode facilitar na transparência e na racionalização dos espaços de pesquisa.

Metodologia: a pesquisa tem uma abordagem qualitativa, realizada por um relatório técnico sobre o processo de implantação do Sistema Integrado de Gestão de Laboratórios de Pesquisa da UFABC. A coleta de dados foi baseada nas técnicas de análise documental e observação participante, ocorridas entre outubro de 2017 e dezembro de 2019, e análise de documentos.

Originalidade/Relevância: esta pesquisa contribui para a discussão sobre a necessidade das instituições de ensino e pesquisa implementarem sistemas integrados de gestão de laboratórios de pesquisa e quais são seus benefícios.

Resultados: os resultados deste estudo demonstraram que a implementação do SIG laboratórios contribuiu para a transparência e racionalização dos espaços de pesquisa dentro da UFABC. Ademais, o sistema passou a ser visto como um ativo estratégico na gestão de bens públicos dentro da universidade.

Contribuições teóricas/metodológicas: A experiência relatada nesta pesquisa apresenta resultados objetivos sobre uma forma estruturada e sistematizada de gestão de laboratórios de pesquisa dentro de uma universidade e induz à reflexão acerca do aperfeiçoamento do modelo de gestão uso do recurso público por meio do SIG.

Contribuições sociais / para a gestão: Este artigo apontou que o sistema de gestão integrada aplicado aos laboratórios de pesquisa pode ser uma ferramenta eficaz para proporcionar o melhor uso aos ativos públicos e também trazer transparência e uso racional dos espaços e recursos para a pesquisa.

Palavras-chave: Sistema Integrado de Gestão; Laboratórios de Pesquisa; Inovação; Gestão de Laboratórios.

\begin{abstract}
Objective: To describe how the Integrated Management System of the research laboratories' implementation process can bring transparency and rationality to the shared Research spaces' usage.

Methodology: The research has a qualitative approach, carried out by a technical report regarding the implementation process of the Integrated Management System for Research Laboratories in UFABC. Data collection was based on the documentary analysis and participant observation techniques, occurred between October 2017 and December 2019.

Originality/Relevance: This research contributes to the discussion about the need of Higher Education School which perform research to implement integrated management systems for research laboratories and what are their benefits.

Results: The obtained results pointed out that implementing Integrated Management Systems was effective on bringing transparency and rationality to manage shared Research spaces in UFABC. Moreover, the system came to be seen as a strategic asset in the management of public goods within the university.

Theoretical/methodological contributions: The experience reported in this research presents objective results on a structured and systematized form of management of research laboratories
\end{abstract}


within a university and induces reflection on the improvement of the management model using the resource through Integrated Management System.

Social/management contributions: This paper pointed out that the integrated management system applied to research laboratories can be an effective tool for providing best usage to the public assets and also bringing transparency and rational usage of the spaces and resources for the research.

Keywords: Integrated Management System; Research Laboratories; Innovation; Laboratory Management.

\section{Resumen}

Objectivo: describir cómo el Sistema Integrado de Gestión del proceso de implementación de los laboratorios de investigación puede aportar transparencia y racionalidad al uso compartido de los espacios de investigación.

Metodología: La investigación tiene un enfoque cualitativo, llevado a cabo por un informe técnico sobre el proceso de implementación del Sistema Integrado de Gestión de Laboratorios de Investigación en UFABC. La recopilación de datos se basó en el análisis documental y las técnicas de observación de los participantes, entre octubre de 2017 y diciembre de 2019.

Originalidad/Relevancia: Esta investigación contribuye a la discusión sobre la necesidad de la Escuela de Educación Superior que realizan investigaciones para implementar sistemas de gestión integrados para laboratorios de investigación y cuáles son sus beneficios.

Resultados: Los resultados obtenidos señalaron que la implementación de Sistemas Integrados de Gestión fue eficaz para aportar transparencia y racionalidad para gestionar espacios compartidos de investigación en la UFABC. Además, el sistema llegó a ser visto como un activo estratégico en la gestión de bienes públicos dentro de la universidad.

Contribuciones teóricas/metodológicas: La experiencia reportada en esta investigación presenta resultados objetivos sobre una forma estructurada y sistematizada de gestión de laboratorios de investigación dentro de una universidad e induce la reflexión sobre la mejora del modelo de gestión utilizando el recurso a través de sistema integrado de gestión.

Contribuciones sociales/de gestión: Este documento señaló que el sistema integrado de gestión aplicado a los laboratorios de investigación puede ser una herramienta eficaz para proporcionar el mejor uso de los activos públicos y también aportar transparencia y uso racional de los espacios y recursos para la investigación.

Palabras clave: Sistema Integrado de Gestión; Laboratorios de investigación; Innovación; Gestión de laboratorio.

\section{Introdução}

O recente cenário brasileiro de inovação e pesquisa é considerado um campo com capacidade para ser explorado. Porém as pesquisas desenvolvidas dentro das universidades são descritas como deficitárias (Lima et. al, 2019). A falta de infraestrutura de um espaço de pesquisa para desenvolvimento de projetos científicos está sendo um dos principais fatores 
inibidores que os pesquisadores enfrentam para condução de pesquisa (Lima et. al, 2019).

No ano de 2004, a Lei de inovação no 10.973 foi aprovada e trata sobre incentivos à inovação e à pesquisa científica e tecnológica no ambiente produtivo. A lei aborda o estímulo que pode ser realizado pela União, os Estados, o Distrito Federal, os Municípios e as agências de fomento. Assim como, fomentar e estimular a formação de alianças estratégicas com os Institutos de Ciência e Tecnologia, nas atividades de pesquisa e a geração de produtos inovadores (Miranda, de Araujo, de Oliveira Freire, \& Fernandes, 2019).

Portanto, para que aconteça a pesquisa alinhada a inovação, ciência e a tecnologia é necessário a integração entre público e privado, por meio da participação direta de empresas privadas ou recursos do sistema público de pesquisa. Para que novas possibilidades de pesquisa se concretizem, essas parcerias precisam ser regulamentadas por resoluções entre as universidades e outras instituições (Carvalho, Aveni, Coimbra, \& Montilha, 2017). A construção de espaços e o desenvolvimento da pesquisa científica são dependentes em sua maioria de fontes não oriundas às instituições e sim das agências de fomento (Junqueira, Bezerra, \& Passador, 2015). Neste contexto, permeia no cenário brasileiro o sistema de produção do conhecimento científico vinculado principalmente ao financiamento público por agências de fomento (Negri \& Cavalcante, 2013; Lima et. al, 2019).

Entretanto, Junqueira, Bezerra e Passador (2015) afirmam que os laboratórios de pesquisa são escassos assim como a dificuldade de desenvolver pesquisa científica no Brasil. Segundo os autores, a dificuldade existe desde a solicitação de financiamento público, análise e aprovação das agências de fomento dos projetos de pesquisa e principalmente ter um laboratório de pesquisa adequado para testar os experimentos e desenvolver o projeto pesquisa.

Embora o financiamento público da infraestrutura de pesquisa confira maior legitimidade às instituições beneficiárias, grupos de pesquisa e acadêmicos, ele traz consigo a necessidade de transparência e uma gestão democrática dos espaços para que todos os pesquisadores tenham condições de trabalho para o desenvolvimento de suas pesquisas (Leal \& Àvila, 2018). É nesse cenário que a Fundação Universidade Federal do ABC percebeu a importância da implantação de um sistema integrado de gestão de laboratórios de pesquisa. A instalação do sistema integrado de gestão de laboratórios de pesquisa foi um anseio da Próreitoria de Pesquisa da UFABC.

Dado esse contexto, a gestão de espaços de pesquisa tornou-se um tema estratégico para as instituições de pesquisa e ensino. Tem-se então como questão de pesquisa entender: como um sistema integrado de gestão de laboratórios de pesquisa da UFABC contribui na 
operacionalização, transparência e otimização dos recursos físicos de pesquisa? O objetivo deste relato técnico é descrever como o processo de implantação do sistema integrado de gestão de laboratórios de pesquisa, pode facilitar na transparência e na racionalização dos espaços de pesquisa.

Para isso, o presente relato técnico encontra-se estruturado em cinco seções, sendo esta introdução a primeira delas. Na segunda seção são abordados os principais conceitos acerca das temáticas: laboratório de pesquisa, inovação organizacional e sistema integrado de gestão. Em seguida, são apresentados os procedimentos metodológicos utilizados no trabalho. Na quarta seção são demonstradas as análises e discussões dos resultados. Por fim, na última seção são expostas as considerações finais, e em sequência as referências bibliográficas utilizadas neste estudo.

\section{Referencial teórico}

Esta seção aprofunda os conceitos que serão utilizados como suporte e embasamento científico, abordando no primeiro momento, um panorama geral sobre o Laboratório de Pesquisa e Inovação Organizacional; e, por fim, Sistema Integrado de Gestão.

\subsection{Laboratório de Pesquisa e Inovação}

Laboratório de pesquisa é descrito como um local provido de infraestrutura para manipulações, exames e experiências efetuados no âmbito da pesquisa científica, de análises e testes técnicos e clínicos e, de ensino que propicia a observação, o experimento e prática sistematizada. Sendo assim, a pesquisa em laboratório traz a ideia de experimento controlado, mas não obrigatoriamente de pesquisa experimental e aplicam-se às ciências exatas, básicas, humanas e de saúde, entre outras (Pagliuca, 2010).

Segundo Teixeira, Nunes e Mello (1998) os laboratórios de pesquisa são formados pela interseção de muitos elementos, como: pesquisadores, técnicos, discentes, auxiliares técnicos, equipamentos e diferentes materiais, cuja unidade é fornecida pelas linhas de pesquisa. Desta forma, para estes autores, os laboratórios de pesquisa podem ser analisados como um campo relacional, formado pela ação conjunta desses atores heterogêneos, caracterizados pela diversidade de interesses e modos de conduta. 
Santarosa, Parisoto e Moreira (2017) afirmam que o desenvolvimento de pesquisa em laboratório é atraente devido às constantes contribuições que podem proporcionar à sociedade. Para lidar efetivamente com o fenômeno da escassez dos espaços de pesquisas, as organizações estão inovando nos modelos de gestão, criando redes ágeis de equipes, implementandofeedback em tempo real através de microaprendizado personalizado (Pelster, Johnson, Stempel, \& van der Vyver, 2017).

A inovação é um componente fundamental no desenvolvimento e geração de valor das instituições, diante disso é necessário elaborar estratégias que identificam e tragam o progresso de gestão. Além do amadurecimento de diferentes formas e com diferentes graus de força, nas quais resulta o caminho estratégico que a instituição deseja (Frezatti, Bido, Cruz, \& Machado, 2015). Lanzer (2012) relatam que a inovação é a transformação do estado das coisas, inserindo alterações que possam ser executadas com sucesso no progresso de novos produtos ou serviços.

A inovação nas Instituições de Ensino Superior proporciona fluxos de transferência de tecnologia para sociedade e outras instituições, o que pode ser impulsionado pela criação de redes de conhecimento e grupo de pesquisas, que isoladamente seria limitado (Herrera, 2017). Desta forma, a inovação organizacional e os laboratórios de pesquisa estão juntamente ligados aos resultados das organizações de pesquisa e a continuidade da sua vantagem competitiva, dada a sua relevância para que sejam executadas de forma satisfatória suas expectativas inovadoras de pesquisa.

\subsection{Sistema Integrado de Gestão}

Sistema Integrado de Gestão (SIG) pode ser conceituado como a compatibilidade de técnicas, processos e procedimentos utilizados em uma organização para instituir suas políticas de gestão e ter um melhor alcance dos objetivos comparando com os sistemas que trabalham de forma isolada (Cicco, 2004).

Pode-se compreender que o SIG como uma ferramenta integradora de processos por meio do compartilhamento de informações, recursos humanos, financeiros e infraestrutura, a fim de satisfazer as necessidades de diferentes partes interessadas (Bernardo, Gotzamani, Vouzas, \& Casadesus, 2018; Kopia, Kompalla, \& Ceausu, 2016). A interação de múltiplos sistemas operacionais permite um recorte não só das competências organizacionais, como também, das responsabilidades dos colaboradores (Bergue, 2012). Neste contexto, o autor relata que a capacitação e conscientização dos colaboradores, responsáveis pela política institucional 
e dos processos da organização, visam comprometimento e planejamento para os rumos almejados organizacionais.

As motivações para a implementação do SIG podem ser intrínsecas e extrínsecas, as motivações intrínsecas são aquelas de razões socioeconômicas de uma organização, que podem resultar de falhas repetidas no cumprimento dos objetivos e metas operacionais, financeiros e/ou regulatórios. Por outro lado, as motivações extrínsecas influenciam a organização a partir do ambiente externo que podem ser devidos à exigência dos cidadãos que obrigam a organização a adotar as melhores práticas de negócios e requisitos regulatórios (Moumen \& Elaoufir, 2018).

Karapetrovic e Casadesus (2009) acreditam que o SIG de qualquer forma deve sempre levar a um sistema mais eficiente. O SIG é considerado um sistema em evolução que corresponde a auto avaliação e benchmarking, sendo que ele fornece orientação e estrutura corporativa e garante que os padrões de certificação individuais sejam atendidos de maneira econômica. Sobre a implantação de um Sistema Integrado de Gestão numa universidade. Moumen e Elaoufir (2018) levantam alguns benefícios da implementação do SIG, conforme a Tabela 1:

\section{Tabela 1}

Benefícios da implementação do SIG

Benefícios da implementação do SIG

- Eliminação da duplicação de documentação

- Redução na duplicação de políticas, procedimentos e registros

- Sistema mais ágil com menos redundância

- Sistemas integrados simplificados, resultando em menos confusão, redundância e conflitos na documentação

- Aumento da eficiência organizacional

- Estrutura comum para melhoria contínua

- Melhoria geral do desempenho organizacional

- Melhorias no gerenciamento de riscos

- Maior satisfação do cliente

- Sistemas simplificados

- Sistema de comunicação aprimorado

- Redução de custos de gerenciamento

\section{Literatura de Apoio}

Douglas e Glen (2000), McDonald et al. (2003), Zutshi e Sohal (2005), Simon e

Douglas (2013), Simon et al. (2014, 2011), Salomone (2008), Santos et al. (2011), Sampaio et al. (2011), Karapetrovic e Casadesus (2009)

McDonald et al. (2003), Simon et al. (2012), Simon e Douglas (2013), Rebelo et al. (2014), Wagner (2007), Hamidi et al. (2012)

Douglas e Glen (2000), Zutshi e Sohal (2005)

Santos et al. (2011), Simon et al. (2011, 2014), Rebelo et al. (2014), Jørgensen et al. (2005), Wright (2000), Douglas e Glen (2000), Zeng et al. (2007), Zutshi e Sohal (2005) e McDonald et al. (2003) 
- Melhorias operacionais

- Meios para o desenvolvimento sustentável

- Economia de tempo

- Maior aceitação pelos funcionários

- Planejamento estratégico

- Visão holistica

- Melhores sinergias entre sistemas

- Melhor utilização dos recursos

- Unificação de auditorias internas

- Redução de custos de auditorias internas

- Melhor uso dos resultados da auditoria

- Unificação de atividades de treinamento

- Economia de recursos humanos

- Melhor definição de responsabilidades

- Melhor alocação de recursos

- Recursos otimizados (financeiros e humanos) para manter um único objetivo

- Promoção de trabalho em equipe

- Trabalhadores mais competentes

- Pessoal mais motivado

- Vantagem competitiva de mercado

- Recursos humanos aumentaram o treinamento dos funcionários

- Otimização / unificação de atividades de treinamento
Fresner e Engelhardt (2004), Holdsworth (2003), Jørgensen et al. (2005), McDonald et al. (2003)

Zutshi e Sohal (2005)

Rocha et al. (2007)

Salomone (2008) e Simon et al. (2011)

Zeng et al. (2007), Salomone (2008), Santos et al. (2011), Abad et al. (2014)

Wright (2000), Salomone (2008), Santos et al. (2011)

Fonte: Moumen, M., \& Elaoufir, H. (2018). An integrated management system: from various aspects of the literature to a maturity model based on the process approach. International Journal of Productivity and Quality Management, 23(2), 218.

Segundo Moumen e Elaoufir (2018) a integração de sistemas de gerenciamento é viável para redução de custos, melhorias operacionais, gerenciamento eficiente e utilização de recursos, motivação dos funcionários e meios para melhorar o cumprimento das obrigações e exigências sociais de diferentes partes interessadas.

Para Botter, Catellino e Itelvino (2012) o sistema integrado de gestão permite o alcance pela otimização e qualidade dos serviços, contínua capacitação profissional e motivação por um comportamento ético e de boas práticas. Os mesmos autores consideram a adoção de práticas gerenciais, apoiadas por sistemas de informação, um diferencial dos novos paradigmas da administração. Nesse contexto, o sistema integrado de gestão torna-se um elemento que impulsiona as instituições a repensarem suas estruturas de funcionamento tradicionalistas.

Segundo Harrington (1993) o aperfeiçoamento e as mudanças organizacionais não acontecem com facilidade e frequência esperadas. É necessário compreender o aperfeiçoamento 
como requisito para desenvolvimento de um ambiente organizacional e, nesse sentido, a implementação do SIG pode auxiliar a melhoria nos processos de gestão (Rebelo, Santos, \& Silva, 2014). Seguindo a mesma compreensão, Nadae (2016) afirma que o SIG encaminha uma série de ganhos organizacionais, na eficácia, na satisfação, no controle, na competitividade na utilização de recursos, na burocracia, na comunicação e principalmente no tempo.

Bernardo, Marti e Stanislav (2009) observaram que as instituições que fazem adesão ao SIG por motivos estratégicos primeiramente e posteriormente partem para os campos das informações tático e operacional. No entanto, é preciso atentar para que o "desenvolvimento de um SIG flexível e aberto às inovações, com capacidade de adaptar-se aos novos paradigmas, valorização do fator humano, dando maior ênfase aos resultados" (Freitas \& Jabbour, 2010, p. 164).

Em relação às dificuldades enfrentadas na implementação do SIG, Simon et al. (2012) verificaram quatro grupos principais de dificuldades, que são: falta de recursos para integração; dificuldades na implementação e certificação de sistemas de gestão de acordo com os padrões; dificuldades internas; e dificuldades de entendimento e uso de normas pelos funcionários. $\mathrm{O}$ primeiro conjunto de dificuldades está relacionado à falta de recursos como capital financeiro, conhecimento, tecnologia, tempo, consultores, diretrizes específicas para integração de sistemas etc. (Simon, Karapetrovic, \& Casadesús, 2012; Bernardo et al., 2012).

Diante dessas impossibilidades, a integração dos sistemas de gestão é realizada de forma limitada, com base nas experiências dos próprios gerentes, que embora comprometidos com o projeto, muitas vezes não possuem o conhecimento necessário para realizar a implementação do SIG, treinam funcionários para conhecer multiplicadores de ponta, criar meios para disseminar a nova cultura SIG na empresa (Asif, Bruijn, Fisscher, Searcy, \& Steenhuis, 2009; Oliveira, 2013).

Quanto ao segundo conjunto, as dificuldades estão relacionadas às diferenças dessemelhanças que ainda existem entre os padrões e que complicam a integração (Abad, Dalmau, \& Vilajosana, 2014). No que diz respeito ao terceiro conjunto, às dificuldades internas, é importante observar que as organizações tradicionalmente mantêm grupos de trabalho separados, e sua integração pode gerar conflitos culturais, resistência à mudança e comunicação (Zeng et al., 2008; Bernardo et al., 2012). Ainda, um último conjunto relacionado às dificuldades decorrentes da falta de motivação, atitude e conhecimento dos funcionários no uso 
dos padrões, também pode afetar a execução das tarefas de forma integrada (Simon, Karapetrovic, \& Casadesús, 2012; Bernardo et al., 2012).

Diante do exposto, acredita-se que no concerne às universidades que desenvolvem pesquisa, a adesão de uma visão sistêmica se torna uma questão necessária, já que os processos da gestão dos dados repercutem na performance na produção científica. Desta forma, entendese a importância da gestão de laboratórios de pesquisa, onde o desempenho é mensurado, apoiado pela tecnologia de informação e pelo alcance da efetividade na produção científica essenciais ao desenvolvimento social e acadêmico-científico.

\section{Procedimentos metodológicos}

O estudo se propôs a investigar a contribuição do SIG laboratórios para a gestão dos laboratórios de pesquisa da UFABC, e para isso a pesquisa realizada tem abordagem qualitativa e tipo exploratória, tendo como objetivo prover o pesquisador de maior conhecimento sobre o tema ou problema de pesquisa em perspectiva, ou ainda quando será preciso conhecer de maneira mais profunda o assunto (Mattar, 2001).

O estudo é de natureza aplicada e foi desenvolvido por meio do levantamento de campo, inicialmente utilizando-se dados secundários (documentos e resoluções relacionados ao tema; mensagens em correio eletrônico trocadas pelas diferentes instâncias de gestão; planos operacionais e das atividades de educação permanente junto aos gestores de unidades e equipes; atas de reuniões de planejamento e pactuação) que foram obtidos em consultas internas dos documentos da Fundação Universidade Federal do ABC e na sua página oficial disponibilizada na internet.

A coleta dos dados foi realizada por meio da técnica de observação participante, as informações foram coletadas no período de outubro de 2017 a dezembro de 2019. A observação participante foi efetuada por um dos autores deste estudo, pois um dos autores, além de desenvolver as atividades técnicas e administrativas na referida instituição, participou de todo o processo de formalização e implementação do SIG laboratórios, conduzida pela Pró-reitoria de Pesquisa da referida universidade, com liderança da Divisão Administrativa de Apoio ao Pesquisador de modo integrado com as Comissões de Pesquisa (Centro de Engenharia, Modelagem e Ciências Sociais Aplicadas - CECS, Centro de Ciências Naturais e Humanas CCNH e Centro de Matemática, Computação e Cognição - CMCC), com o apoio técnico do Núcleo de Tecnologia da Informação. 
A técnica ora mencionada é um método que possibilita ao pesquisador, participar do fenômeno e, desse modo, desenvolver um relacionamento de confiança, necessário para revelar "os bastidores das realidades" de sua experiência (Paterson, Bottorff, \& Hewat, 2003). Assim, foi possível descrever a partir não só do acompanhamento, mas de ações ocorridas durante todo o processo. Fato que, inclusive, respalda a justificativa desta pesquisa. A análise dos dados compreendeu dois tipos de análise. Primeiramente, procurou examinar todos os documentos produzidos pela UFABC, que foram deliberadas para institucionalização e o funcionamento dos laboratórios de pesquisa, o SIG laboratórios e os dados dos laboratórios cadastrados no SIG laboratórios. O segundo esforço foi direcionado revelar os bastidores, por meio da observação participante e rela, do contexto da implementação do SIG laboratórios e sua utilização.

\section{Resultados obtidos e análise}

Nesta seção se desenvolve uma apresentação e a análise e discussão dos resultados. Especificamente, são apresentadas, a UFABC e a caracterização do problema, e por fim, a implementação do SIG laboratórios.

\subsection{Caracterização da Organização e do problema analisado}

A UFABC é uma instituição federal de ensino superior (IFES) e a discussão de sua criação ocorreu no Congresso Nacional em 2004, a instituição foi criada por meio do projeto Lei $\mathrm{n}^{\mathrm{o}} 3.962 / 2004$ de iniciativa do poder executivo, e aprovado como a Lei $\mathrm{n}^{\mathrm{o}} 11.145$ no dia 26 de julho de 2005. A referida instituição foi proposta em um Programa de governo que buscou remodelar as políticas públicas de educação no Brasil por intermédio de um novo plano de desenvolvimento da educação e programas como o Prouni, que teve papel fundamental na ampliação de vagas nas IFES, e o REUNI de 2007, o Programa de Apoio a Planos de Reestruturação e Expansão das Universidades Federais, o qual ofereceu condições para as universidades federais aumentarem o acesso e a permanência na educação superior. Assim, a UFABC se insere num contexto de grandes mudanças estruturais e pedagógicas nas IFES.

Criada pela Lei $\mathrm{n}^{\circ} 11.145$, de 26 de julho de 2005, e alterada pela Lei ${ }^{\circ} 13.110$, de 25 de março de 2015, que definiu a dotação de personalidade jurídica de direito público, a UFABC é uma fundação pública criada para atender principalmente a demanda da região do $\mathrm{ABC}$ paulista (formado pelos municípios de Santo André, São Bernardo do Campo, São Caetano, Diadema, Mauá, Ribeirão Pires e Rio Grande da Serra), bem como outras localidades de 
interesse institucional, criando um ambiente favorável ao desenvolvimento social, já que, segundo a referida lei, a UFABC “terá por objetivo ministrar educação superior, desenvolver pesquisa nas diversas áreas do conhecimento e promover a extensão universitária, caracterizando sua inserção regional mediante atuação multicampi na região do ABC paulista, bem como em outras localidades de interesse institucional”.

Nesse sentido, a UFABC tem um modelo de projeto pedagógico diferenciado, pois apresenta características interdisciplinares que pretendem auxiliar sua comunidade com ensino, pesquisa e extensão de excelência, buscando o aprimoramento da ciência, tecnologia e inovação. Sua criação visou também a atender uma demanda de ensino superior público e gratuito, dado que a região do $\mathrm{ABC}$ paulista não possuía instituições de ensino superior com perfil semelhante.

Para tanto, de acordo com suas normas, a UFABC é regida pela legislação federal pertinente e conta com autonomia administrativa, didático-científica, gestão financeira e disciplinar e uma estrutura de dois campi, Santo André e São Bernardo do Campo, órgãos superiores da administração, órgãos setoriais e de apoio acadêmico e complementar.

Os setores administrativos e acadêmicos da UFABC, com seus quadros de servidores, prestam os variados serviços demandados pela clientela da universidade. Dentre as áreas e setores que compõem a administração da UFABC, destacam-se a Reitoria, três centros (Centro de Engenharia, Modelagem e Ciências Sociais Aplicadas - CECS, Centro de Ciências Naturais e Humanas - CCNH e Centro de Matemática, Computação e Cognição - CMCC), interdisciplinares, sete Pró-Reitorias, a Secretaria Geral, a Prefeitura Universitária, a Agência de Inovação, o Núcleo de Tecnologia da Informação, e a Superintendência de Gestão de Pessoas, entre outros.

Já quanto aos seus servidores, a UFABC informa que, em janeiro de 2019, eles se encontravam divididos da seguinte forma: 794 docentes, lotados nos três centros, responsáveis pelo ensino, pesquisa e extensão desenvolvidos pela instituição, além de ocuparem, eventualmente, cargos administrativos de direção (como chefes, coordenadores, diretores, reitor, pró-reitores, entre outros); e 726 técnicos-administrativos distribuídos pelos diversos setores e áreas da universidade, sendo 483 de cargos de nível médio (classe D) e 243 de cargos de nível superior (classe E).

Os laboratórios de pesquisa da UFABC, conforme informado pela instituição, em novembro de 2019, conta com a disponibilidade de 204 instalações, sendo que estão divididos em três categorias: a) laboratórios dos grupos de pesquisa (LGP); b) laboratórios multicentros 
(LMC) e c) laboratórios multiusuários (LMU). Os LMU são espaços de pesquisa e desenvolvimento sob administração direta da Pró-Reitoria de Pesquisa que tem características interdisciplinares e multiusuária. Vários campos do conhecimento científico podem utilizar esta mesma infraestrutura para desenvolver pesquisa, sendo estes laboratórios dentro da UFABC: a Central Experimental Multiusuário (CEM) e suas expansões e anexos, e os Biotérios. Os LGP são espaços temáticos de pesquisa e desenvolvimento outorgados à administração direta de um dos Centros, enquanto os LMC são espaços temáticos de pesquisa e desenvolvimento outorgados à administração conjunta de mais que um Centro.

Os laboratórios da universidade são temáticos, e não podem ser considerados espaços pessoais ou exclusivos. Com a devida justificativa, e com a anuência explícita do coordenador (caso dos LMU) ou do docente responsável (caso dos LMC e LGP), todos os docentes da UFABC poderão utilizar os equipamentos disponíveis em qualquer laboratório da UFABC, independentemente da alocação do docente e do projeto, e da fonte de recursos utilizada para a aquisição do equipamento. Todos os LGP, LMC e LMU deverão passar por avaliações periódicas, para verificar a produtividade dos mesmos e justificar o uso e concessão do espaço. A definição dos critérios e prazos da avaliação da produtividade, bem como a publicação dos seus resultados, deverá ser realizada pelas Comissões de Pesquisa dos Centros (caso dos LGP e LMC) ou pela Pró-Reitoria de Pesquisa (caso dos LMU). Laboratórios que não atendam aos critérios serão considerados como disponíveis.

Foi constatado que desde a criação da UFABC até a implementação do SIG laboratórios de pesquisa existiam problemáticas sobre informações ausentes, não padronizadas e duplicadas sobre os laboratórios de pesquisa da referida instituição, o que ocasionava alocamentos de colaboradores de forma desorganizada, desproporcional e pouco conhecimento de infraestrutura adequado em face aos tipos de pesquisas desenvolvidas.

Face às exposições acima e as dificuldades encontradas na gestão de laboratórios de pesquisa da Universidade Federal do ABC (UFABC), o módulo de Laboratórios do Sistema Integrado de Gestão (SIG) foi desenvolvido e implementado, com o intuito de disponibilizar aos gestores da instituição uma plataforma de gestão com acesso rápido e simplificado para inserção e coleta de informações pertinentes à infraestrutura, localização, colaboradores, tipo de pesquisa desenvolvida, entre outros tópicos referentes aos laboratórios de pesquisa, contribuindo, dessa forma, para uma gestão de laboratórios eficiente, eficaz e transparente. A 
Tabela 2 apresenta os principais documentos que resultaram na implementação do SIG laboratórios:

\section{Tabela 2}

Principais documentos para a implementação do SIG laboratórios

\section{Documento}

(“Lei no 10.973”, 2004)

(“Lei no 11.145”, 2005)

(“Decreto no 5563”, 2005)

(“Lei no 13.110", 2015)

(“Lei no 13.243”, 2016)

("Resolução ConsUni n 84", 2012)

("Resolução ConsUni n 106", 2013)

("Resolução ConsUni nº185", 2018)

("Resolução ConsUni nº187", 2018)

("Resolução ConsUni nº 193", 2019)

("Resolução ConsEPE n¹27", 2012)

Planos Diretores de Tecnologia da Informação - PDTIC da UFABC

\section{Descrição}

Dispõe sobre os incentivos à inovação e a pesquisa científica e tecnológica e sua regulamentação;

Cria a UFABC e Institui a Fundação Universidade Federal do ABC UFABC e dá outras providências

Regulamenta a Lei n. 10.973 que dispõe sobre incentivos à inovação e à pesquisa científica e tecnológica no ambiente produtivo, e dá outras providências.

Altera a Lei no 11.145 , de 26 de julho de 2005, que institui a Fundação Universidade Federal do ABC - UFABC, e dá outras providências

Dispõe sobre estímulos ao desenvolvimento científico, à pesquisa, à capacitação científica e tecnológica e à inovação (novo marco de CT\&I)

Cria o Comitê Estratégico de Tecnologia da Informação e Comunicação (CETIC) da UFABC

Cria e dispõe sobre as Comissões de Pesquisa dos Centros da Fundação Universidade Federal do ABC.

Dispõe sobre o Comitê Estratégico de Tecnologia da Informação e Comunicação (CETIC) e inclui dentre suas competências as tarefas de Comitê de Governança Digital, revoga e substitui a Resolução ConsUni $n^{\circ} 84$

Dispõe sobre o Comitê Estratégico de Tecnologia da Informação e Comunicação (CETIC), revoga e substitui a Resolução ConsUni n ${ }^{\circ}$ 185.

Institui a Comissão de Pesquisa (CoPes) da UFABC.

Regulamenta a classificação e as normas para alocação dos laboratórios de pesquisa da UFABC

Instrumentos que permitiram nortear e acompanhar a atuação das

Tecnologias da Informações da Comunicação - TIC na UFABC, definindo estratégias e o plano de ação para implementá-las.

Fonte: Elaborado pelos autores (2019).

A UFABC regulamentou suas atividades de gestão laboratorial e a implementação do Sistema Integrado de Gestão de Laboratórios por meio das resoluções do Conselho Universitário - ConsUni, do Conselho de Ensino, Pesquisa e Extensão - ConsEPE e dos planos

Diretores de Tecnologia da Informação - PDTIC que delegou as competências e diretrizes para 
a atuação da Pró-Reitoria de Pesquisa e as Comissões Permanentes de Pesquisa do CECS, CCNH e CMCC.

Inicialmente, desde a criação da UFABC no ano de 2005, a universidade não possuía um sistema integrado de gestão de laboratórios, sendo estas mapeadas por planilhas eletrônicas não padronizadas, o que dificultava ações de extração de dados necessários para tomada de decisões dos gestores e proporcionava imprecisões das informações e duplicação de dados, o que criava vários desajustes para alocação de pessoal, infraestrutura e insumos para os laboratórios de pesquisa. Desta maneira, existia uma necessidade de implementar um sistema integrado para uma melhor gestão.

\subsection{Implementação do Sistema Integrado de Gestão}

Durante a $1^{\text {a }}$ fase da implementação do SIG na UFABC no ano de 2013, o PDTIC realizou estudos iniciais sobre melhorias dos sistemas institucionais de gestão, para auxiliar nestes objetivos foram criados grupos de trabalho com a finalidade de verificar qual o sistema mais adequado para gestão acadêmica e administrativa para instituição. Os grupos de trabalhos indicaram em seus relatórios finais que o SIG desenvolvido pela Universidade Federal do Rio Grande do Norte - UFRN era o que atendia as especificidades, sendo firmado um convênio de cooperação entre UFABC e UFRN no ano de 2014 para aquisição do referido sistema.

Somente no ano de 2017 foi disponibilizado SIG laboratórios que permite a inserção de informações referente à infraestrutura de pesquisa, pessoal alocado, além de integrar informações dos sistemas patrimônio (equipamentos e mobiliários) e projetos de pesquisa.

Durante a $2^{a}$ fase, a Pró-Reitoria de Pesquisa verificou através da atualização do SIG que foi implementado no começo de 2017 todas as funcionalidades, percebendo que o referido sistema atenderia perfeitamente a gestão dos laboratórios, pois o sistema permitia a inserção de coleta de informações pertinentes à infraestrutura, localização, colaboradores, tipo de pesquisa desenvolvida, entre outros tópicos referentes aos laboratórios de pesquisa (Figura 1). 
10 Fase

Disponibilidade do módulo laboratório

Disponibilidade do SIG

28. Fase

Reunião com o Núcleo de Tecnologia da Informação (NTI)

$3^{2}$ Fase

Teste do módulo laboratório

4 Fase

Criação dos Manuais

50 Fase

Reuniões com as partes interessadas

6. Pase

Conscientização, divulgação e orientação do módulo laboratório de pesquisa para comunidade acadêmica

Inserção dos dados no SIG laboratórios

8: Fase

Divulgação dos dados no SIG Público

9 Fase

Figura 1. Fases para implementação do SIG laboratórios Fonte: Elaborado pelos autores (2019).

Logo em seguida, a $3^{\mathrm{a}}$ fase aconteceu o agendamento de reuniões entre PROPES e o NTI para verificar a possibilidade de realizar testes preliminares e possibilidade de implementação do sistema, logo após o NTI deu o aval para realizar os testes e implementação $\left(4^{\mathrm{a}}\right.$ fase $)$. 


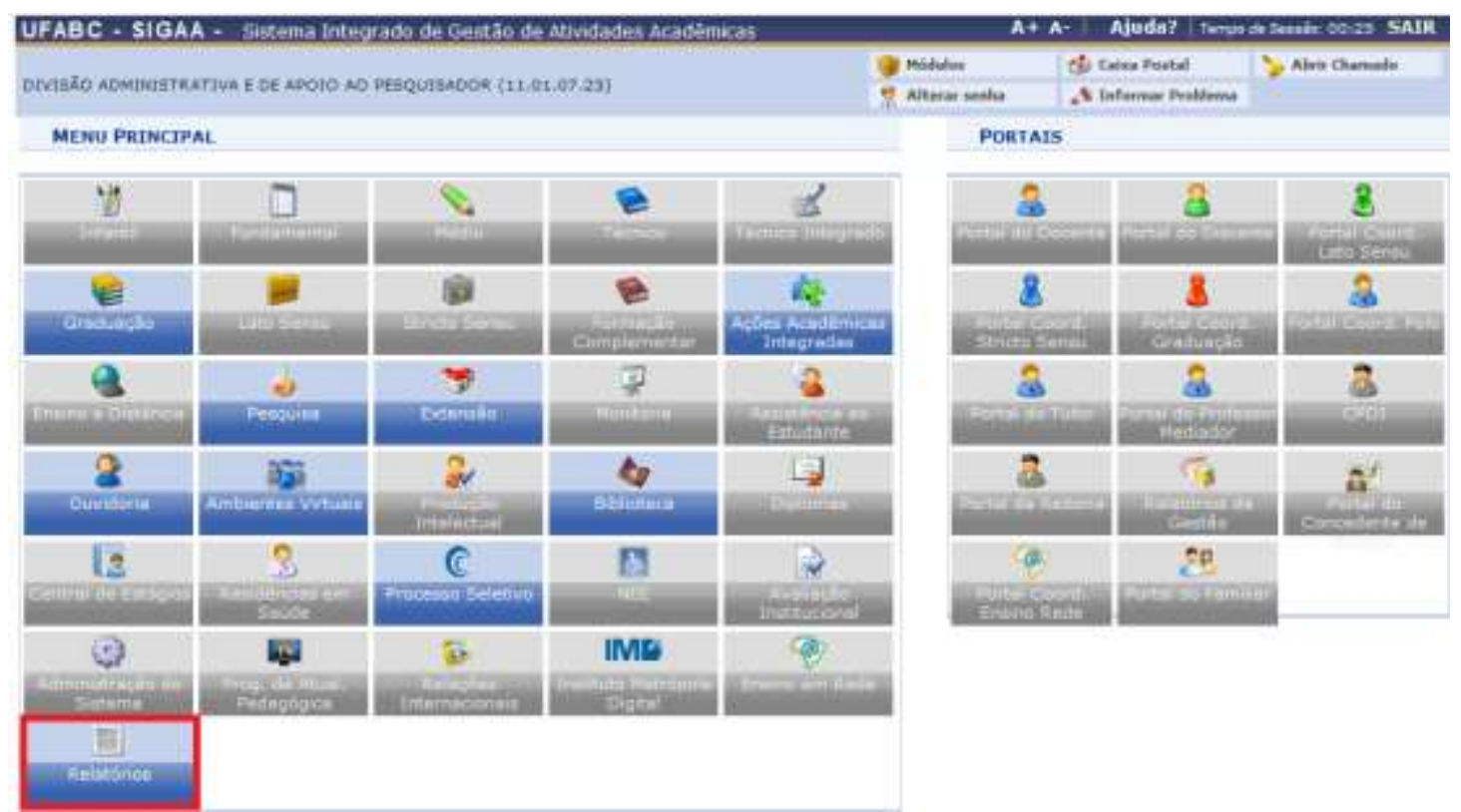

Figura 2. Funções do SIG - UFABC

Fonte: Universidade Federal do ABC (2019)

Finalizados os testes, a $5^{\text {a }}$ fase consistiu na elaboração de dois manuais para que os usuários do sistema tivessem um material de apoio para inserção e gestão das informações, o primeiro manual, intitulado "Manual do usuário do SIG laboratórios" orienta de forma bem didática os coordenadores dos laboratórios em como inserir as informações, conforme exigências do sistema, o segundo manual, intitulado "Manual do gestor do SIG laboratórios" foi desenvolvido para auxiliar os gestores da PROPES e dos Centros em como realizar a gestão do SIG laboratórios, além de validar as informações fornecidas pelos coordenadores, dessa forma, realizando um controle a posteriori da informações fornecidas pelos coordenadores (Figura 2).

Posteriormente a $6^{\mathrm{a}}$ fase, a PROPES realizou reuniões com os representantes das partes interessadas do SIG laboratórios (CCNH, CMCC e CECS) para apresentar o SIG laboratórios e os manuais, onde foram ouvidas as sugestões e encaminhados ao NTI para melhoria do sistema, só com a concordância do produto final do sistema foi realizado o lançamento do sistema.

A $7^{\text {a }}$ fase foi o lançamento, conscientização da importância e divulgação do SIG laboratório de pesquisa, onde foi divulgado via e-mail para comunidade acadêmica sobre SIG laboratório de pesquisa e disponibilidade para treinamento pela PROPES a toda comunidade. $\mathrm{Na} 8^{\mathrm{a}}$ fase aconteceu a inserção dos laboratórios pelos coordenadores e validação pelos gestores 
dos laboratórios e por fim, a $9^{a}$ fase foi a divulgação a qualquer cidadão dos laboratórios de pesquisa pelo SIG público.

A Figura 3 demonstra o sistema interno onde os gestores da UFABC podem gerar relatórios das atividades tanto em formato de planilha eletrônica e/ou em formato de texto.

\begin{tabular}{|c|c|c|c|}
\hline \multirow[b]{2}{*}{ DIVISȦO ADMTNISTRATIVA E DE APOIO AO PESQUISADCR $(12,01,07.23)$} & PI Módulos: & (1) Caixa Postal & \multirow[t]{2}{*}{ 'y Abrir Chamado } \\
\hline & 8 Alterar senha & A Informar Problems & \\
\hline \multicolumn{4}{|c|}{ LISTA DE RELATORIOS } \\
\hline \multicolumn{4}{|l|}{ Titulo } \\
\hline Coordenadores de projetos de pesquisa & & $\begin{array}{l}\text { Gerar } \\
\text { arquivo } \\
\text { Microsoft } \\
\text { Excel xis }\end{array}$ & 圈 \\
\hline E-mails dos alunos ativos para questionários & & $\begin{array}{l}\text { Gerar } \\
\text { arquivo } \\
\text { Microsoft } \\
\text { Excel XLS }\end{array}$ & 卓 \\
\hline Tipo de ensino médio dos alunos - Graduaçâo & & $\begin{array}{l}\text { Gerar } \\
\text { arquivo } \\
\text { Microsoft } \\
\text { Excel XLS }\end{array}$ & 器 \\
\hline Laboratónios de Pesquisa & & $\begin{array}{l}\text { Gerar } \\
\text { arquivo } \\
\text { Microsoft } \\
\text { Excel XIS }\end{array}$ & 望 \\
\hline Quantidade de disciplinas cursadas/Aluno/Quad & & $\begin{array}{l}\text { Gerar } \\
\text { arquivo } \\
\text { Microsoft } \\
\text { Excel Xis }\end{array}$ & 亜 \\
\hline E-mails dos servidores ativos para questionários & & $\begin{array}{l}\text { Gerar } \\
\text { arquivo } \\
\text { Microsoft } \\
\text { Excel Xis }\end{array}$ & 氜 \\
\hline
\end{tabular}

Figura 3. Relatório de pesquisa gerados pelo SIG - UFABC Fonte: Universidade Federal do ABC (2019)

Enquanto a Figura 4 mostra telas de páginas web onde é possível que qualquer usuário com acesso a internet acesse as informações dos laboratórios de pesquisa da UFABC, dessa forma, colaborando para visualização do recorte de infraestrutura de pesquisa e as pesquisas desenvolvidas nestes espaços, tomadas de decisões racionalizadas da alocação de pessoal e equipamento e principalmente para transparência. 


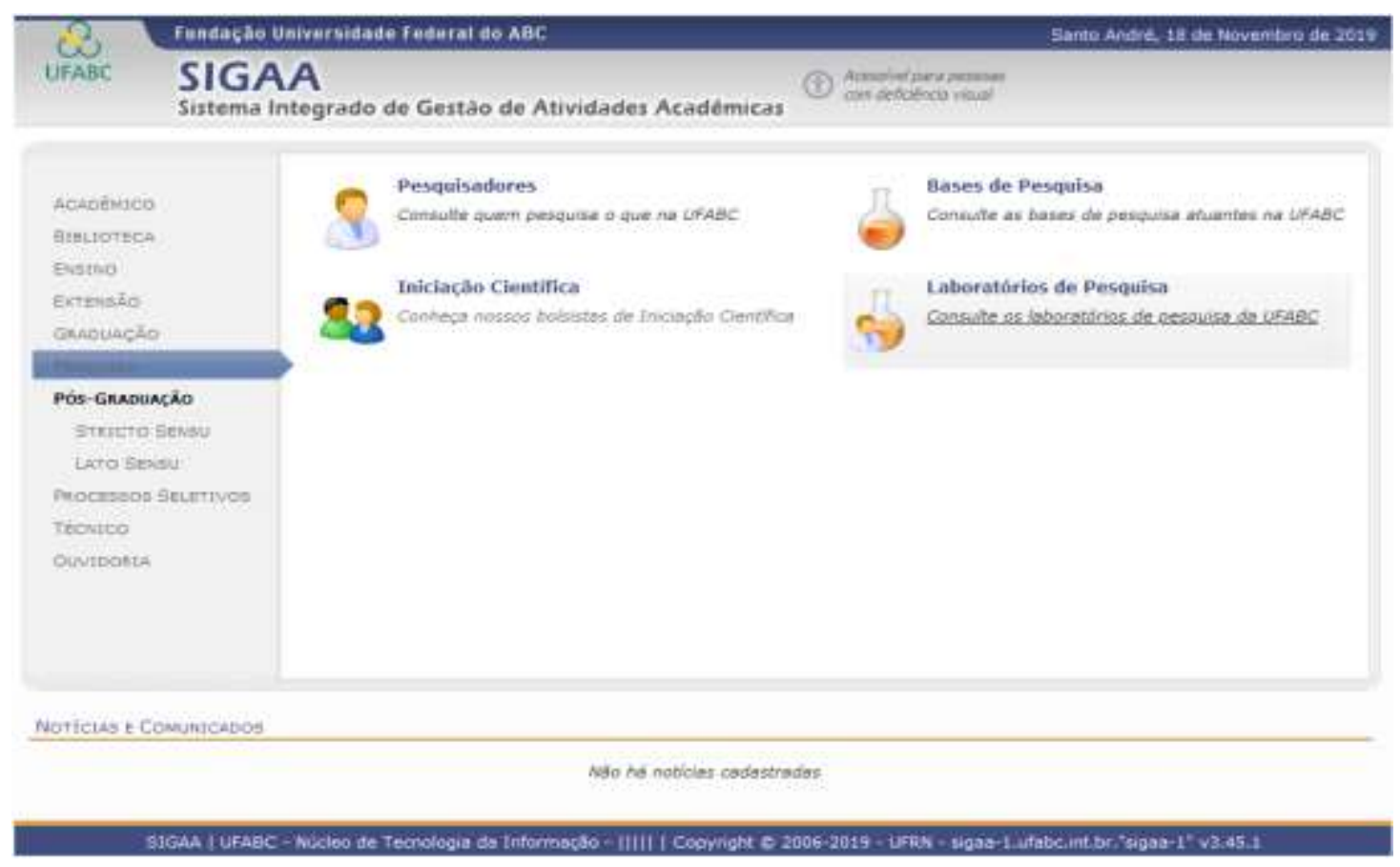

Figura 4. Acesso do usuário ao SIG - UFABC

Fonte: Universidade Federal do ABC (2019)

O planejamento e o SIG laboratórios foram fundamentais para inovação gestão de laboratórios, características para implementação do SIG. Sobretudo, praticando uma política institucional de gestão de dados de recursos de infraestrutura de pesquisa. Além da preocupação constante com os envolvidos nos processos da organização e, por fim, resultando transparência nos laboratórios da UFABC.

Pode-se afirmar que, no SIG laboratórios, a vantagem competitiva se dá pelo auxílio para coleta de dados e a facilidade da divulgação das informações (Figura 5), pois um sistema padronizado, único e sincronizado permite que os dados coletados sejam fidedignos e que colabora para as tomadas de decisões sobre de alocação dos recursos de pessoal e equipamentos sejam mais coerentes e democráticas. Portanto, o sistema tornou-se um ativo estratégico para a gestão de bens públicos dentro da universidade. 


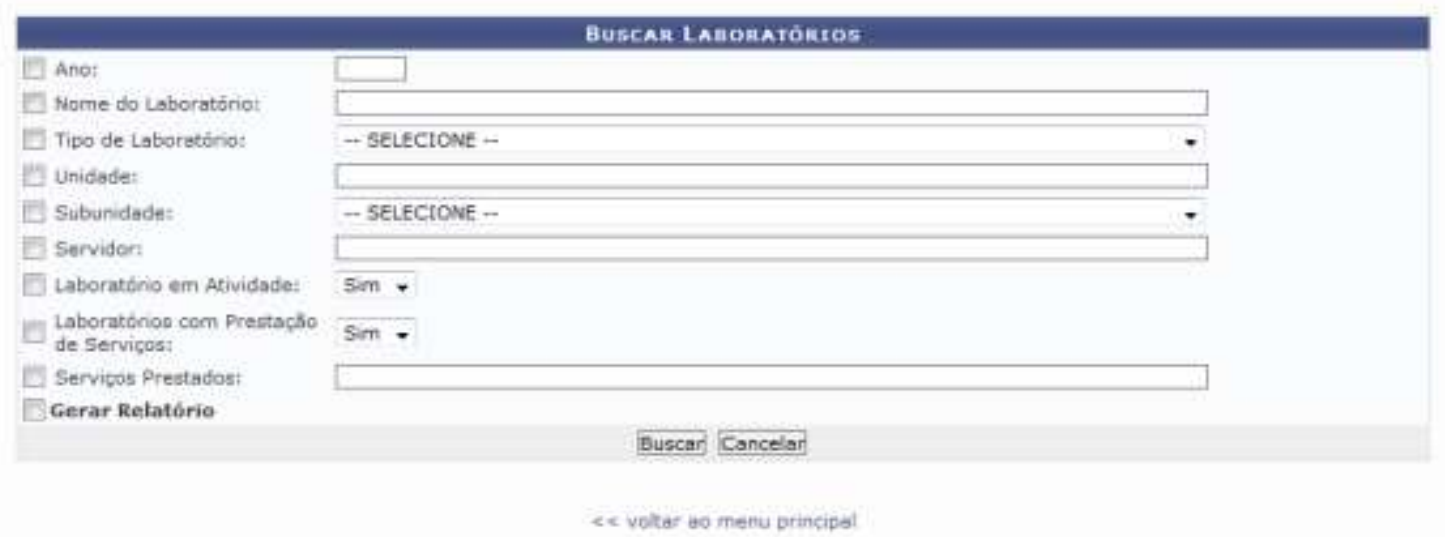

Figura 5. Acesso ao Público SIG - UFABC

Fonte: Universidade Federal do ABC (2019)

O estudo vai ao encontro à posição do SIG como inovação, ao afirmar que SIG laboratórios é considerado consequência dos processos e ações da instituição, orientada para criar um sistema integrado, que possibilita a criação do valor agregado, coerente com o suporte teórico dos autores Moumen e Elaoufir (2018), que discutem os benefícios do SIG para as diferentes partes interessadas. O SIG laboratórios, como instrumento, permite o alcance pela otimização e qualidade dos serviços, contínua capacitação profissional e motivação por um comportamento ético e de boas práticas, que conforme Nadae (2016) traz diversos ganhos organizacionais.

Mas a gestão dos laboratórios só surtirá efeito se a organização dispuser do monitoramento das forças intervenientes no seu ambiente operacional como descrevem Freitas e Jabbour (2010), para fomentar a adaptabilidade aos paradigmas, o ser humano e os resultados. Esse raciocínio corrobora a inevitável inter-relação entre os coordenadores de laboratórios e os gestores da UFABC, caso os coordenadores não estejam comprometidos em fornecer os dados e os gestores não monitorar as informações e motivar os coordenadores no fornecimento dos dados nada adianta ter um sistema que facilite os dados se os interessados não querem passar as informações. 


\section{Considerações finais}

Pode-se concluir com o estudo que a UFABC adotou a gestão de dados de laboratórios de pesquisa através do SIG laboratórios, procurando diferenciar-se das demais instituições de pesquisa e tirando o máximo proveito de otimização, racionalização e de apoio relacionados à infraestrutura de pesquisa.

A análise documental possibilitou verificar como funcionam os laboratórios de pesquisa e o SIG laboratórios, além de identificar que antes da adoção do SIG laboratórios a instituição tinha problemas para levantamento da infraestrutura de pesquisa. A observação participante auxiliou em identificar o processo de implementação que não foram encontrados na documentação. Também, corroborou com o entendimento da política institucional da gestão de laboratórios da instituição.

Neste relato é destacada a disponibilização do SIG laboratórios na UFABC que ajudou a construir uma nova cultura, pois proporcionou transparência e os conteúdos desenvolvidos nos laboratórios estão para a sociedade, entretanto esbarrou em alguns costumes e práticas, como a aderência e conscientização de alguns coordenadores de laboratórios em disponibilizar os dados do laboratório de pesquisa com frequência. Reconhecendo as dificuldades de controles e acompanhamentos dos dados e informações produzidas pela UFABC, o compromisso institucional com a implantação do SIG-UFABC está presente nas diversas ações que estão correlacionadas, buscando o sucesso na implantação nestes primeiros anos de experiência.

O resultado deste estudo demonstra que a implementação do SIG laboratórios contribuiu para inovação tecnológica na gestão de dados da infraestrutura de pesquisa, e que, trata-se de um sistema estratégico que precisa ter o apoio institucional e das instâncias superiores para regulamentar as suas atribuições, além de uma equipe estável formada por profissionais qualificados e especializados, que acumulem experiência a fim de darem continuidade no sistema, mesmo ocorrendo mudanças na equipe gestora (comum no setor público), e que possam assegurar o cumprimento das políticas e diretrizes institucionais.

Apesar de os resultados apontarem para que o SIG contribua para gestão dos laboratórios, a falta de envolvimento dos colaboradores é um grande limitador para coleta e disseminação das informações, nada adianta, ter um sistema ágil e completo, se os coordenadores dos laboratórios não se sintam comprometidos em repassarem as informações ao sistema. 
A partir das acepções ora discutidas acredita-se que o SIG, pelas características aqui apresentadas, é opção, se bem utilizado, de práticas de eficiência, eficácia, efetividade e economicidade, fato que se supõe pode proporcionar resultados benéficos para a comunidade acadêmica e a sociedade. Por fim, espera-se que este trabalho contribua para a área de gestão de laboratórios no Brasil, por meio da demonstração da implementação e operacionalização do SIG para gestão dos laboratórios e transparência dos laboratórios de pesquisa, em conjunto com pesquisas qualitativas e quantitativas que possam ampliar a discussão dos resultados obtidos.

\section{Referências}

Abad, J., Dalmau, I., \& Vilajosana, J. (2014). Taxonomic proposal for integration levels of management systems based on empirical evidence and derived corporate benefits. J. Clean. Prod. 78, 164-173.

Asif, M., de Bruijn, E. J., Fisscher, O. A., Searcy, C., \& Steenhuis, H. J. (2009). Process embedded design of integrated management systems. International journal of quality \& reliability management. 26, 261-282.

Bergue, S. T. (2012). Cultura e mudança organizacional. 2. ed. Florianópolis: Departamento de Ciências da Administração/UFSC, Brasília: CAPES - UAB.

Bernardo, M., Gotzamani, K., Vouzas, F., \& Casadesus, M. (2018). A qualitative study on integrated management systems in a non-leading country in certifications. Total Quality Management \& Business Excellence, 29(3-4), 453-480.

Bernardo, M., Casadesus, M., Karapetrovic, S., \& Heras, I. (2012). Do integration difficulties influence management system integration levels?. Journal of cleaner production, 21(1), 2333.

Bernardo, M., Casadesus, M., \& Karapetrovic, S. (2009). Are methods used to integrate standardized management systems a conditioning factor of the level of integration? An empirical study. International journal for quality research, 5(3), 213-222.

Botter, R. C., Catellino, G. G., \& Itelvino, L. S (2012). Os fatores críticos de sucesso para a implantação de sistemas integrados de gestão no setor público. Anais do Simpósio de Excelência em Gestão e Tecnologia, Resende, RJ, Brasil.

Carvalho, S. M. S., Aveni, A., Coimbra, L. M., \& Montilha, H. F. D. (2017). Empreendedorismo, tecnologia e inovação: Temas contemporâneos na gestão da Universidade de Brasília. Cadernos de Prospecção, 10(4), 626.

Cicco, F. (2004). Sistemas integrados de gestão: agregando valor aos sistemas ISO 9000, Centro de Qualidade, Segurança e Produtividade, São Paulo.

Freitas, W. R. S., \& Jabbour, C. J. C (2010). Rumo à gestão estratégica de Recursos Humanos: estudo de caso em uma organização pública paulista. Revista de Ciências da Administração, 12 (26), p. 163-188.

Frezatti, F., Bido D. S., Cruz, A. P. C., \& Machado, M. J. C. (2015). A estrutura de artefatos de controle gerencial no processo de inovação: existe associação com o perfil estratégico?. Brazilian Business Review, 12(1), 129.

Harrington, H. J. (1993). Aperfeiçoando processos empresariais. São Paulo: Makron Books.

Herrera, D. H. P. (2017). Mecanismos de transferência de tecnologia nas universidades tecnológicas: comparação do modelo brasileiro e colombiano (Dissertação de Mestrado, Universidade Tecnológica Federal do Paraná). 
Junqueira, M. A. D. R., Bezerra, R. C. R., \& Passador, C. S. (2015). O escritório de gestão de projetos de pesquisa como uma inovação organizacional nas universidades. Revista GEINTEC-Gestão, Inovação e Tecnologias, 5(1), 1835-1849.

Karapetrovic, S., \& Casadesus, M. (2009) 'Implementing environmental with other standardized management systems: scope, sequence, time and integration', International Journal of Cleaner Production, Vol. 17, No. 5, pp.533-540.

Kopia, J., Kompalla, A., \& Ceausu, I. (2016). Theory and practice of integrating management systems with high level structure. Quality-Access to success, 17(155), 52-29.

Lanzer, E. (2012). O processo de inovação nas organizações do conhecimento. Florianópolis: Pandion.

Leal, J. C., \& Ávila, M. L. D. (2018). Desestruturação do Modelo de Gestão Compartilhada do FNDCT e a Crise do Apoio Público à Pesquisa Científica e Desenvolvimento Tecnológico no Brasil. I Seminário de Avaliação de Políticas de CT\&I.

Lima, L. A., Oliveira, L. P., da Silva Tenório, L. X., Py-Daniel, S. S., Fernandes, T. L., Ghesti, G. F., \& da Silva, M. L. (2019). Desenvolvimento tecnológico e a maturidade das pesquisas no âmbito das instituições de pesquisa científica e tecnológica-icts no brasil. Cadernos de Prospecção, 12(1), 31.

Mattar, F. N. (2001). Pesquisa de marketing (Vol. 3). São Paulo: Atlas.

Miranda, A. L. B. B., de Araujo, I. T., de Oliveira Freire, B. G., \& Fernandes, A. J. (2019). Inovação nas universidades: uma análise do novo marco legal. Revista ENIAC Pesquisa, 8(1), 85-98.

Moumen, M., \& Elaoufir, H. (2018). An integrated management system: from various aspects of the literature to a maturity model based on the process approach. International Journal of Productivity and Quality Management, 23(2), 218. doi:10.1504/ijpqm.2018.089157

Nadae, J. (2016). Sistemas de gestão integrados como indutor para a sustentabilidade: uma análise do impacto no desempenho das organizações baseado no Triple Bottom Line. (Tese de doutorado). Universidade de São Paulo, São Paulo.

Negri, F., \& Cavalcante, L. R. (2013). Sistemas de Inovação e Infraestrutura de Pesquisa: considerações sobre o caso brasileiro. Brasília: IPEA. 2013, 11 p.

Oliveira, O. J. (2013). Guidelines for the integration of certifiable management systems in industrial companies. Journal of Cleaner Production, 57, 124-133.

Pagliuca, L. M. F. (2010). Reflexões sobre laboratório de pesquisa. Revista da Rede de Enfermagem do Nordeste, 11(4).

Paterson, B. L., Bottorff, J. L., \& Hewat, R (2003). Blending observational methods: possibilities, strategies and challenges. International Journal of Qualitative Methods, Edmonton, 2(1), p. 29-38, 2003.

Pelster, B., Johnson, D., Stempel, J., \& van der Vyver, B. (2017). Careers and learning: Real time, all the time. 2017 Global Human Capital Trends.

Rebelo, M. F., Santos, G., \& Silva, R. (2014). A generic model for integration of quality, environment and safety management systems. The TQM Journal.

Santarosa, M. C., Parisotto, M., \& Moreira, M. A. (2017). Observações em um laboratório de supercondutividade e magnetismo: aspectos metodológicos e epistemológicos. Revista do professor de Física. Revista do Professor de Física, Brasília, 1(1) , p 1-20.

Simon, A., Karapetrovic, S., \& Casadesús, M. (2012). Difficulties and benefits of integrated management systems. Ind. Manag. Data Syst. 112, 828-846.

Teixeira, M. D. O., Nunes, T., \& Mello, J. M. C. D. (1998). Trabalho técnico em laboratórios de pesquisa e desenvolvimento em saúde: um estudo de caso. Hist. ciênc. saúdeManguinhos, 4(3), 493-512. 
Universidade Federal do ABC (2019). Sistema Integrado de Gestão.

Universidade Federal do ABC (2019). Sistema Integrado de Gestão Público.

Zeng, S. X., Tam, V. W., \& Tam, C. M. (2008). Towards occupational health and safety systems in the construction industry of China. Safety science, 46(8), 1155-1168. 